\title{
Meiotic nondisjunction and sperm aneuploidy in humans
}

\author{
D loannou ${ }^{1,2}$, J Fortun ${ }^{3}$ and H G Tempest ${ }^{1,4}$ \\ ${ }^{1}$ Department of Human and Molecular Genetics, Herbert Wertheim College of Medicine, Florida International \\ University, Miami, Florida, USA, ${ }^{2}$ IVF Florida Reproductive Associates, Margate, Florida, USA, ${ }^{3}$ Department of \\ Cellular Biology and Pharmacology, Herbert Wertheim College of Medicine, Florida International University, Miami, \\ Florida, USA and ${ }^{4}$ Biomolecular Sciences Institute, Florida International University, Miami, Florida, USA
}

Correspondence should be addressed to H G Tempest; Email: htempest@fiu.edu

\begin{abstract}
Infertility is relatively common affecting approximately 1 -in- 6 couples. Although the genetic basis of infertility is increasingly being uncovered, the contribution of male infertility often remains unexplained. The leading cause of pregnancy loss and cognitive impairment in humans is chromosome aneuploidy. Sperm aneuploidy is routinely evaluated by fluorescence in situ hybridization. The majority of studies have reported similar findings, namely: (1) all men produce aneuploid sperm; (2) certain chromosomes are more prone to undergo chromosome nondisjunction; (3) infertile men typically have significantly higher levels of sperm aneuploidy compared to controls and (4) the level of aneuploidy is often correlated with the severity of the infertility. Despite this, sperm aneuploidy screening is rarely evaluated in the infertility clinic. Within recent years, there appears to be renewed interest in the clinical relevance of sperm aneuploidy. We shall examine the gender differences in meiosis between the sexes and explore why less emphasis is placed on the paternal contribution to aneuploidy. Increased sperm aneuploidy is often perceived to be modest and not clinically relevant, compared to the female contribution. However, even small increases in sperm aneuploidy may impact fertility and IVF cycle outcomes. Evidence demonstrating the clinical relevance of sperm aneuploidy will be discussed, as well as some of the challenges precluding widespread clinical implementation. Technological developments that may lead to widespread clinical implementation will be discussed. Recommendations will be suggested for specific patient groups that may benefit from sperm aneuploidy screening and whether preimplantation genetic testing for aneuploidy should be discussed with these patients.

Reproduction (2019) 157 R15-R31
\end{abstract}

\section{Introduction}

Primary infertility in humans is relatively common affecting around $15 \%$ of couples trying to conceive (De Kretser 1997). In some instances, mechanical and physiological defects are identified (e.g., tubal blockages, disturbances in the HPG axis etc.). However, the underlying cause of infertility in couples often remains unexplained (Hotaling \& Carrell 2014). A diagnosis of unexplained infertility is often difficult for patients and leaves limited options for treatment, the majority of which relies on expensive and emotionally draining assisted reproductive technology (ART). Male factors contribute to approximately $50 \%$ of cases, solely or in combination with female factors (Chandra et al. 2005). In some instances, perturbations in the male partner are identified such as abnormal semen parameters. In many instances, the cause of reduced semen parameters fails to be identified (e.g., CFTR mutations that lead to absence of the vas deferens causing obstructive azoospermia). Despite the fact that male factors significantly contribute to infertility, the clinical work-up and available diagnostic tests for male factor infertility is underwhelming, with much of the clinical and diagnostic work-up focusing on the female partner. In males, the work-up is usually restricted to family history, physical exam and assessment of semen parameters as per the WHO guidelines (World Health Organization 2010, loannou et al. 2016). Current testing in the male partner is only able to determine the underlying cause of the infertility in around $20 \%$ of cases, which leaves around $80 \%$ of male factor infertility unexplained (Hotaling \& Carrell 2014). Assessment of semen parameters provides some fundamental information and may indicate the need for further genetic testing (e.g. karyotyping, CFTR mutation analysis and/or $\mathrm{Y}$ chromosome microdeletion analysis). However, the clinical validity of the semen assessment has been strongly questioned (Lewis 2007, Hotaling \& Carrell 2014). Furthermore, none of the aforementioned tests assesses the genetic integrity of a semen sample (loannou et al. 2016). Several tests that assess the genetic integrity of sperm have been developed and are available clinically, including tests that evaluate levels of DNA fragmentation, epigenetics and sperm aneuploidy. Sperm aneuploidy has been assessed using the technically challenging human sperm hamster oocyte fusion assay and more recently 
by fluorescence in situ hybridization (FISH) (Fig. 1) (Shi \& Martin 2000, Tempest \& Griffin 2004, Templado et al. 2013). Despite their availability, the clinical utilization of these tests has been very low. In this review, we shall discuss the gender differences in meiosis and the role of aneuploidy in infertility, focusing on the paternal contributions to aneuploidy.

The paternal contribution to aneuploidy is often overlooked in the ART field and is rarely considered clinically relevant. However, we shall present data that suggest increased sperm aneuploidy may negatively impact fertility and ART outcomes and can result in aneuploid offspring. We will explore the challenges to widespread clinical application of sperm aneuploidy screening, identify patients who may benefit the most from screening and potentially preimplantation genetic testing for aneuploidy (PGT-A). In addition, we shall discuss future technological advancements that may lead to widespread clinical implementation of sperm aneuploidy screening.

\section{The paternal contribution to infertility is frequently overlooked}

The sperm cell is an essential prerequisite to fertilization and embryogenesis. Nevertheless, our understanding of the role of sperm in embryogenesis
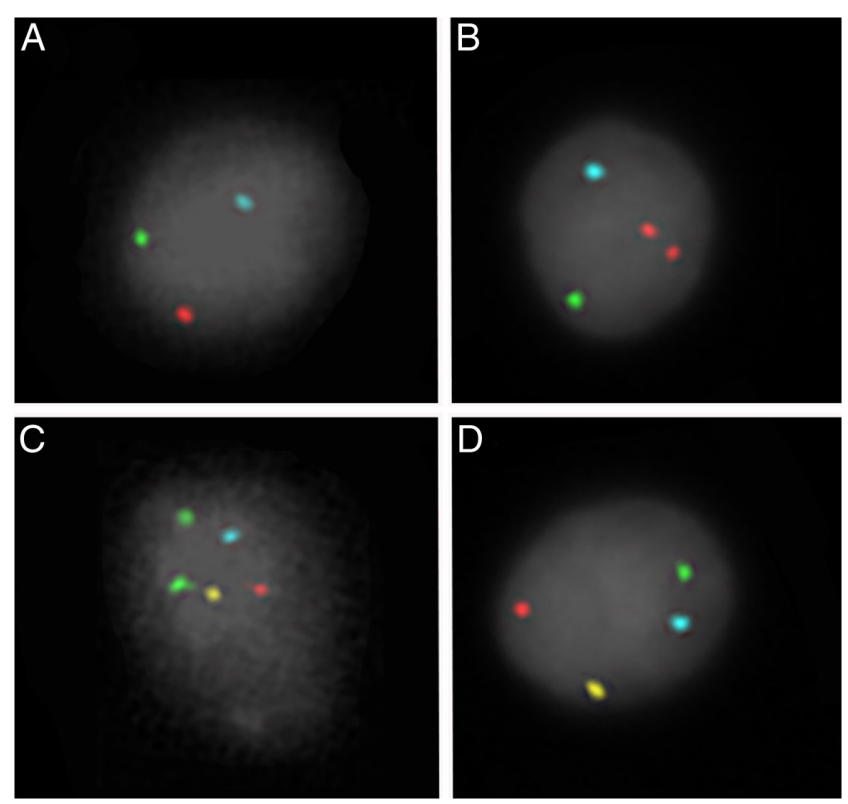

Figure 1 Fluorescence in situ hybridization (FISH) to detect sperm aneuploidy. FISH in human sperm nuclei utilizing FISH probes against chromosomes 18 (aqua), 21 (green), $X$ (gold) and $Y$ (red). Sperm nuclei are DAPI counterstained and have been pseudo colored gray to facilitate visualization of FISH probes. Panels A, B, C and D provide examples of normal and aneuploid sperm for the aforementioned chromosomes tested. Panel A: normal haploid $Y$ bearing sperm; B: YY disomy; C: disomy 21 and XY disomy; and D: $\mathrm{XY}$ disomy. is poorly understood and largely overshadowed by the maternal oocyte (Ioannou \& Tempest 2015). The gametes differ in terms of their known functions in these processes, which likely explains why there is a focus on the maternal rather than the paternal contribution. Following fertilization, the oocyte remodels the paternal genome, replacing the protamines with maternally derived histones and repairing any DNA damage to the paternal genome (Mclay \& Clarke 2003). Moreover, the oocyte drives embryogenesis by contributing the cytoplasmic environment, enzymes, energy and the majority of support organelles required for the first embryonic divisions (Carrell 2013). Thus, spermatozoa are often considered to be a silent vessel whose sole function is to deliver the haploid paternal genome to the haploid oocyte. The relative perceived insignificance of spermatozoa when compared to the oocyte may, in part, explain the lack of interest to develop sperm tests and the scarcity of relevant clinically validated tests for male factor infertility (Kavitha \& Malini 2014).

\section{Chromosome aneuploidy and its relationship to infertility}

Increasingly, genetic factors are being identified as the underlying basis of infertility. Perhaps one of the best known genetic factors that clearly contribute to infertility is chromosome aneuploidy. Chromosome aneuploidy is the presence of extra or missing chromosomes from the normal chromosome complement and is the leading cause of pregnancy loss and cognitive impairment in humans. Interestingly, the prevalence of chromosome aneuploidy seems to be largely restricted to humans. Thus, we do not have adequate animal models to study the mechanisms leading to a high incidence of chromosome aneuploidy in humans. The vast majority of chromosome aneuploidies in humans are not viable in a non-mosaic state and frequently lead to spontaneous abortions, often before a clinical pregnancy is even realized. Although several chromosomes $(13,18,21, \mathrm{X}$ and $\mathrm{Y})$ are potentially viable in an aneuploid state, a significant proportion of these are also spontaneously aborted (Nagaoka et al. 2012). The most established risk factor for aneuploid conceptions is advanced maternal age (AMA) which is defined as women aged 35 years or older. The clinical relevance of the paternal contribution to chromosome aneuploidy is frequently overlooked, despite the fact that aneuploid conceptuses are paternally derived in 5-10\% of autosome aneuploidies and $5-100 \%$ of aneuploidies involving the sex chromosomes (Hassold et al. 1993). This is likely due to the milder phenotypes seen in sex chromosome aneuploidies compared to other viable chromosome aneuploidies. However, there remains a risk of spontaneous abortion, mild cognitive impairment, infertility, and other phenotypes associated with sex chromosome aneuploidies, as well as more severe 
clinical phenotypes for autosomal aneuploidies that are paternally derived.

\section{Meiosis: differences between males and females}

There are fundamental biological differences between meiosis in males and females, particularly regarding timing and outcome. In females, meiosis begins in utero, arrests during prophase I at around 20 weeks of gestation, and only resumes when oocytes are selected from the pool for ovulation following the onset of puberty. Meiosis I in females is only completed in ovulated follicles, and meiosis II is only completed if the ovulated oocyte is fertilized. In contrast, males undergo the process of spermatogenesis, in which spermatogonial stem cells differentiate into spermatozoa over a period of 74 days (Samplaski et al. 2010). In males, spermatogonia undergo constant mitotic proliferation at the onset of puberty and these primordial germ cells differentiate, undergo meiosis without arrest and eventually form mature spermatozoa. Another significant difference between males and females is found in the number of haploid gametes produced at the end of meiosis. Meiosis results in the generation of four haploid gametes in males, but only one haploid gamete and two polar bodies in females. Moreover, there is a progressive and significant atresia of oocytes throughout in utero development until menopause in females, whereas in males there is constant proliferation of spermatogonial stem cells following puberty, which leads to the production of millions of sperm each day throughout their lifetime. Based on the intrinsic differences between meiosis in males and females, it is perhaps not surprising that chromosome aneuploidy in gametes is higher in females than in males and that the risk for aneuploid offspring is associated with AMA, given that oocytes have been arrested in meiosis I for decades. The postulated mechanisms of chromosome nondisjunction have been extensively reviewed elsewhere (Nagaoka et al. 2012, Webster \& Schuh 2017).

\section{Meiotic studies}

Given the biological differences between males and females, it stands to reason that the biological mechanisms that go awry leading to the generation of chromosomally aneuploid gametes are likely to be different. Studies evaluating chromosome pairing and meiotic recombination during the prophase I stage of meiosis in males and females are predominantly performed using immunofluorescence staining. Typically, fluorescent antibodies against SCP1 and SCP3 of the synaptonemal complex are used, which detects proteins involved in the pairing of homologous chromosomes, antibodies against MLH1 detects sites of meiotic recombination, and CREST antibodies detect chromosome centromeres (Fig. 2). Some studies have utilized FISH following immunofluorescence to identify individual chromosomes. For the most part, human studies have primarily focused in males due to the difficulty in obtaining female meiotic preparations. Because studies in males require an invasive testicular biopsy, the majority of published data come from infertile patients with either obstructive- or nonobstructive azoospermia (NOA) undergoing a testicular biopsy as part of ART treatment, or patients with presumably normal spermatogenesis, but undergoing a vasectomy reversal. Thus, the data obtained in these studies may not be entirely representative of the normal testicular environment.
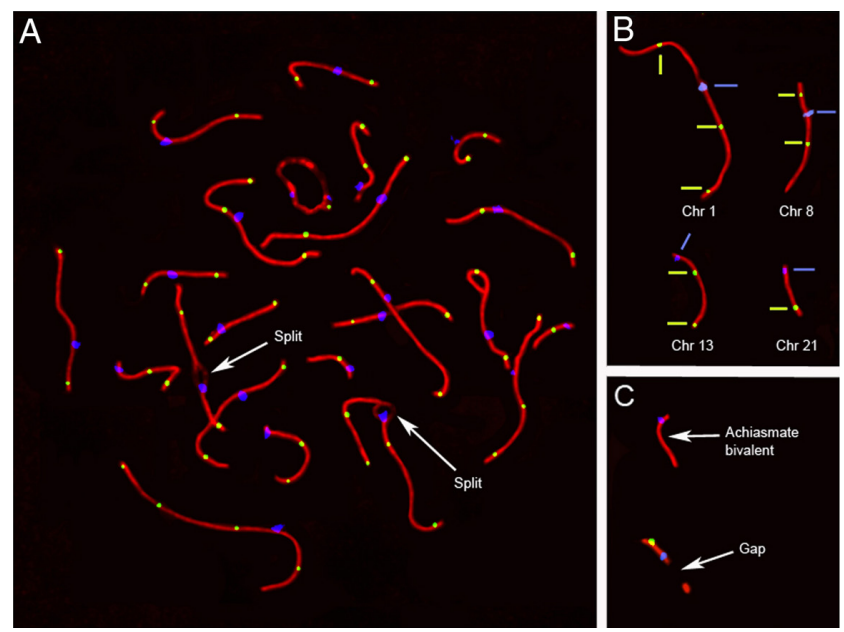

Figure 2 Immunofluorescence of human pachytene spermatocytes. Chromosome synapsis is detected using antibodies against the synaptonemal complex (SCP1 and SCP3) labeled in red and sites of meiotic recombination by antibodies against MLH1 labeled in yellow. In addition, CREST antibodies labeled in blue are used to detect chromosome centromeres. Panel A displays a complete pachytene spermatocyte spread, note synaptonemal pairing defects resulting in splits in the synaptonemal complex (indicated by arrows). In panels B and C individual bivalents from pachytene spreads are displayed. Panel B includes bivalents from four different chromosomes $(1,8,13$, and 21). MLH1 recombination foci are indicated by yellow lines to the left of the synaptonemal complex and the location of the centromere of each chromosome is indicated by the blue line to the right of each synaptonemal complex. Note the differences in synaptonemal complex length between the chromosomes going from the largest (chromosome 1) to the smallest (chromosome 21). The larger the chromosome the more sites of recombination, with chromosome 21 possessing a single recombination foci. Each $\mathrm{p}$ - and $\mathrm{q}$-arm possesses at least one site of recombination (chromosomes 13 and 21 are acrocentric chromosomes so do not have a p-arm), and we can see evidence of positive interference of recombination (foci are not located in close proximity to each other). Panel C displays two bivalents with meiotic defects indicated by arrows, the top bivalent is achiasmate (lacking recombination) and the bottom bivalent has a defect in chromosome pairing resulting in a gap in the synaptonemal complex. Images provided by Renee H Martin. 


\section{Lessons learned from meiotic prophase I studies}

Several key consistent themes have emerged from various published studies investigating chromosome pairing and meiotic recombination in prophase I (Tempest 2011). Studies of male and female meiotic preparations reiterate the differences between sexes. Females possess more sites of recombination than males ( 70 vs $\sim 50$, respectively per meiotic spread) (Gruhn et al. 2013). The number of recombination foci per chromosome is correlated with chromosome size; in essence, the larger the chromosome the more sites of recombination. For example, chromosome 1 is the largest and contains an average of five recombination sites, whereas chromosome 21 is the smallest and has a single recombination site in males (Martin 2008, Sun et al. 2005, 2008). In addition to chromosome size, gene density may also play a role. For example, even though chromosomes 18 and 19 are similar in size, they differ dramatically in gene density (Boyle et al. 2001, Babariya et al. 2017). Chromosome 19 is one of the most gene-rich chromosomes, whereas chromosome 18 is one of the gene poorest chromosomes. Chromosome 19 has a longer synaptonemal complex, and consequently, more recombination foci on average when compared to chromosome 18 in males (Sun et al. 2004b). With the exception of the acrocentric chromosomes, the $\mathrm{p}$ - and $q$-arms of all chromosomes possess at least one site of recombination in the normal situation (Hassold et al. 2000). There is also clear evidence of suppression of recombination in close proximity to other sites of recombination (Fig. 2) (Sun et al. 2004b). Furthermore, the preferential localization of sites of recombination along the synaptonemal complex demonstrates a more distal localization in males, when compared to females (Gruhn et al. 2013).

\section{The relationship between perturbations in male meiosis and infertility}

Clear differences in meiosis are observed between fertile and infertile men. The vast majority of meiotic preparations in fertile men tend to be in the pachytene stage, since this is the longest stage of meiotic prophase I (Gonsalves et al. 2004). This is in sharp contrast to many infertile patients. For example, NOA patients tend to have significantly reduced numbers of cells in the pachytene stage or complete absence of pachytene cells due to a partial or complete block at the zygotene stage of meiosis (Gonsalves et al. 2004). Additionally, a significant reduction or lack of recombination foci (achiasmate bivalents) (Fig. 2) has been observed in some NOA patients compared to fertile controls (Ferguson et al. 2007, Sun et al. 2008). FISH studies following immunofluorescence have reported that achiasmate bivalents most frequently involve chromosomes 21, 22, X and Y (Ferguson et al. 2007,
Sun et al. 2008). In males, these four chromosomes typically possess a single site of recombination, whereas all other chromosomes possess two or more. Meiotic recombination may be crucial to prevent the premature separation of homologous chromosomes, which can lead to chromosome nondisjunction in gametes. If this were the case, it would suggest the chromosomes that possess a single site of recombination have the highest risk to be achiasmate, and thus, the highest rates of chromosome nondisjunction, compared to chromosomes with multiple sites of recombination. Indeed, several studies have identified a correlation between the presence of achiasmate bivalents in NOA patients and increased levels of sperm aneuploidy, predominantly involving the aforementioned chromosomes (Ferguson et al. 2007, Sun et al. 2008). This finding is further corroborated by the majority of sperm FISH studies, which report significantly higher levels of sperm aneuploidy for these chromosomes (21, $22, X$ and $Y$ ) in both fertile and infertile subjects (Shi \& Martin 2000, Tempest \& Griffin 2004, Templado et al. 2013). Sequencing of single spermatozoa also demonstrated a lower frequency of recombination in aneuploid sperm (Lu et al. 2012). Indirect evidence of the association between aberrant recombination and sperm aneuploidy was also provided by determining the parental origin of trisomic conceptuses and liveborns (Hassold et al. 1993). This study demonstrated that the majority of paternally derived $47, \mathrm{XXY}$ offspring lacked recombination at either the p- or q-arm pseudoautosomal regions of the sex chromosomes (Hassold et al. 1993). Additional evidence to suggest lack of recombination may be a contributing factor to aneuploidy is also suggested from SNP array data in cleavage-stage embryos (Rabinowitz et al. 2012).

In addition to disturbances in recombination, perturbations in chromosome pairing have also been observed between fertile and infertile patients. The incidence of synaptonemal complex pairing disturbances is more frequently observed in infertile than fertile patients (Sun et al. 2004a, 2007, Martin 2006). These pairing disturbances can include gaps (absence of synaptonemal complex formation in regions) or splits (formation of the synaptonemal complex, but failure of the synaptonemal complex to join) (Fig. 2), all of which may contribute to chromosome nondisjunction. It is important to note that meiotic studies in infertile men are usually restricted to patients with azoospermia. Azoospermia is a rare cause of male infertility and represents the most severe category of male infertility; thus, the discussed findings may not be widely applicable in patients with milder semen parameter disturbances. Nevertheless, evidence of the contribution of achiasmate bivalents and chromosome aneuploidy has been observed in multiple studies using various techniques in both fertile and infertile patients, cleavage-stage embryos and aneuploid offspring. This suggests that the production 
of achiasmate bivalents is strongly associated with an increased risk of chromosome nondisjunction.

\section{Mechanisms of chromosome aneuploidy in males}

The underlying mechanism(s) for increased aneuploidy observed in some men remains to beelucidated. However, defects in the meiotic machinery leading to chromosome nondisjunction have been identified in some patients. This includes perturbations in chromosome pairing and recombination (e.g., achiasmate bivalents) and/or defects in an as of yet unidentified meiotic checkpoint that prevents aneuploid sperm from completing meiosis (Harton \& Tempest 2012). Evidence of a male meiotic checkpoint comes from studies that have assessed the sperm aneuploidy frequencies in $47, \mathrm{XXY}$ and $47, \mathrm{XYY}$ patients. In the absence of gonadal mosaicism, the expected frequency of sex chromosome aneuploidies in the sperm of these patients would be $50 \%$; however, studies report significantly lower levels of sperm aneuploidy (1-25\%) in these patients, concomitant with a general reduction in sperm count (Ferlin et al. 2005, Harton \& Tempest 2012). These studies provide indirect evidence that there is likely a 'leaky' meiotic checkpoint that prevents the completion of meiosis for a significant proportion of aneuploid sperm, and may also contribute to the low sperm counts in these patients. The existence of a meiotic checkpoint in males is further supported by studies showing that the frequency of aneuploidy in meiosis II spermatocytes is $14 \%$, similar to the levels reported in young females (Garcia-Cruz et al. 2010, Uroz \& Templado 2012). This finding suggests that the differences in aneuploidy levels found in gametes between males and females may have less to do with differences in chromosome nondisjunction, but rather, could be due to a meiotic checkpoint that may be more efficient in males than in females (Andreescu et al. 2016).

Despite the known genetic contribution, the hunt to identify the genes and mutations that may contribute to male infertility and increased sperm aneuploidy remains elusive. Studies including genome-wide association studies have been largely unsuccessful in implicating the genes involved or reproducing study findings, suggesting that male infertility is likely attributed to rare, low-frequency variants of small effect size, precluding their identification (Hotaling \& Carrell 2014). Given the considerable phenotypic heterogeneity of male infertility, the complexity of spermatogenesis, the more than 2300 genes involved and the likely multifactorial mode of inheritance, it is perhaps not surprising that we have been unsuccessful in identifying SNPs, copy number variants $(\mathrm{CNVs})$ or mutations associated with male infertility (Hotaling \& Carrell 2014). In summary, we have a very limited understanding of the genetic mechanisms contributing to male infertility and increased sperm aneuploidy.

\section{Human sperm aneuploidy}

\section{The advantages and disadvantages of FISH to assess sperm aneuploidy frequencies}

Prior to the widespread use of FISH, a few studies utilized the technically challenging and laborious approach of karyotyping sperm chromosomes through the sperm hamster oocyte fusion assay. Thus, the vast majority of studies have predominantly utilized FISH to assess sperm aneuploidy levels. Prior to assessing the use of FISH to assess sperm aneuploidy frequencies, it is beneficial to review some of the major advantages of FISH that led to its widespread for molecular cytogenetic applications. FISH is a pivotal cytogenetic tool used to detect genetic abnormalities that has been used by researchers and clinical diagnostic labs worldwide for decades. FISH has a high sensitivity and specificity if appropriate probes are selected and applied to relevant target cells or tissues. FISH has several key advantages: (1) it is relatively rapid to perform, (2) it can be performed in interphase cells and thus, it does not require metaphase chromosomes, (3) it can multiplexed by utilizing multiple fluorochromes up to five (or more when fluorochromes are used in combination) and (4) robust high-throughput automated systems exist for some FISH applications, which permit automated slide loading, image capture, and analysis with minimal user intervention. These advantages led to the widespread use of this very versatile molecular cytogenetic tool for a wide variety of research and clinical diagnostic applications.

Despite the numerous advantages of FISH, there are several major drawbacks for the utilization of FISH to assess sperm aneuploidy. Due to the lack of commercially available FISH probes in different fluorochromes, the majority of sperm FISH studies only utilize three to five probe targets at any one time. Furthermore, sperm aneuploidy screening requires the use of a strict scoring criteria and unlike the majority of other FISH applications, the accurate assessment of sperm aneuploidy requires the scoring of thousands of sperm nuclei due to its relatively low incidence. Therefore, sperm aneuploidy screening is laborious and costly in terms of technician time, and precludes analysis of semen samples in patients with very low sperm counts. Although FISH probes are commercially available for a wide variety of targets, they can be costly on a per sample basis, especially for less commonly used fluorochromes as these may need to be custom made. The cost is potentially increased further due to the need of a larger probe volume (compared to other FISH applications) to score the large number of cells. Typically, aneuploidy screening for three to five chromosomes may cost patients in the region of $\$ 500-700$, which also precludes the widespread implementation of this test clinically. The vast majority of sperm FISH aneuploidy studies often restrict the chromosomes analyzed to those involved in clinically viable chromosome aneuploidies 
(chromosomes 13, 18, 21, X and Y). Consequently, evaluation of sperm aneuploidy by FISH only assesses a handful of chromosomes and does not provide a genome-wide assessment of aneuploidy for all 24 chromosomes in a single cell. In order to expand to more than five targets in a single experiment, FISH probes need to be mixed with different ratios of fluorochromes, which can be detected by specialized software used for spectral karyotyping applications; yet, this is not widespread amongst laboratories. Moreover, as FISH is performed in small highly condensed interphase nuclei assessing increasing targets is challenging due to the risk of overlapping signals and inability to rapidly score nuclei manually. Typically, studies that assess more than five chromosomes achieve this by performing additional rounds of FISH. Due to the thousands of sperm nuclei scored, it is technically challenging to re-probe the same sperm nuclei to increase the number of chromosomes assessed in each cell. One feasible approach to bypass many of these challenges is to utilize automated highthroughput systems.

To date, we and others largely agree that automated systems can reliably assess sperm aneuploidy (Netten et al. 1997, Baumgartner et al. 2001, Perry et al. 2007, 2011, Carrell \& Emery 2008, Molina et al. 2009, Tempest et al. 2010, Martinez et al. 2013, Branch et al. 2017). Some studies have reported substantial reductions in analysis time (Branch et al. 2017), but it is important to consider some challenges. One challenge is the unique highly condensed chromatin packaging found in sperm cells, which sees the majority of histones replaced with protamines. This unique packaging requires sperm cells to be treated with dithiothreitol, lithium diiodosalicylate, pepsin or heparin to facilitate access of FISH probes to the DNA (this step is not required in any other cell type). An additional challenge is that FISH signals are often found on very different focal planes, requiring the capture of multiple Z stacks for each fluorochrome. Of note, a similar challenge is faced by manual scoring, requiring the scorer to carefully examine multiple focal planes for each cell. Thus, some studies have noted that the analysis time for automated systems may not be reduced per se, when compared to manual scoring. However, manual intervention is significantly less, and restricted to performing the FISH and reviewing aneuploid cells (Molina et al. 2009, Tempest et al. 2010). Careful attention is required for slide preparation (e.g., sperm density) and to optimize the software to exclude cells that do not fulfill the scoring criteria used by highly trained cytogeneticists (e.g., overlapping cells and various FISH signal criteria) (Molina et al. 2009, Tempest et al. 2010). This may in part explain some of the differences reported for some samples between manual and automated scoring (Molina et al. 2009, Tempest et al. 2010). However, once established and validated, automated approaches can be used to score more cells, chromosomes and patients to achieve increased statistical power and importantly reduce costs (Branch et al. 2017). Automated systems are widely used in high-throughput diagnostic labs particularly in the field of oncology. The major limitation for its use to evaluate sperm aneuploidy is cost, and the requirement for high sample volumes to warrant purchasing such a system. Thus, most published studies have utilized manual scoring. With increasing clinical demand for sperm aneuploidy testing, however, it is likely that the use of automated approaches will increase. In the next sections, we will focus on the clinical relevance of sperm aneuploidy testing, and which patients would most likely benefit.

\section{Sperm aneuploidy levels in men with proven fertility or with normal semen parameters}

Over 50 publications have demonstrated that all men produce a proportion of aneuploid sperm (Shi \& Martin 2000, Tempest \& Griffin 2004, Templado et al. 2013). In males with proven fertility or normozoospermia (semen parameters within the normal WHO reference ranges), extrapolations from tested chromosomes suggest that approximately $3-5 \%$ of sperm are aneuploid (Pang et al. 1999, Tempest \& Griffin 2004, Lu et al. 2012). If we look at chromosomes individually, around $0.1-0.15 \%$ of sperm are aneuploid for each of the 24 chromosomes (Pang et al. 1999, Shi \& Martin 2000, Tempest \& Griffin 2004). However, chromosomes such as $21,22, X$ and $Y$ have a two- to three-fold higher incidence of aneuploidy than the other chromosomes, with a frequency of around $0.3 \%$ per chromosome (Pang et al. 1999, Shi \& Martin 2000, Tempest \& Griffin 2004). Studies have shown that sperm aneuploidy frequencies are largely consistent over time in fertile or normozoospermic males with little intraindividual variation (Robbins et al. 1993, Rademaker et al. 1997, Rubes et al. 2005, Tempest et al. 2009). However, significant differences in sperm aneuploidy can occur at single time points in some individuals, suggesting aneuploidy frequencies can be altered, perhaps by transient factors, lifestyle changes or environmental exposures (e.g., diet, infection, exercise, smoking or air pollution etc.) (Tempest et al. 2009). Furthermore, there are clearly interindividual differences, with some males consistently producing higher levels of sperm aneuploidy (Rademaker et al. 1997, Rubes et al. 2005, Tempest et al. 2009).

To date, a handful of studies have examined whether advancing paternal age contributes to sperm aneuploidy. The majority of these provide little evidence to support a correlation between advancing paternal age and increased levels of sperm aneuploidy (Fonseka \& Griffin 2011, Andreescu et al. 2016, Donate et al. 2016). There may be a very small effect for a handful of chromosomes in much later years, typically decades after what would traditionally be considered 'reproductive age' (Griffin et al. 1995, Fonseka \& Griffin 2011, Garcia- 
Ferreyra etal. 2018). However, as spermatogonia undergo mitotic proliferation, they are prone to replicative errors such as insertions and deletions, SNPs, point mutations and epigenetic alterations. As sperm cells differentiate, there is limited DNA repair, and thus, a higher risk of transmitting alterations to future offspring. A link between advanced paternal age and increased risk of sperm aneuploidy has not been established (most likely due to the biological differences in meiosis between males and females). Nevertheless, increasing evidence suggests an association with advanced paternal age and an increased risk of single-gene and multifactorial disorders (e.g., achondroplasia, apert syndrome, autism, and schizophrenia amongst others) (Yatsenko \& Turek 2018).

\section{Sperm aneuploidy as a biomarker to monitor environmental and occupational exposures}

Multiple studies have demonstrated an association between environmental factors and increased sperm aneuploidy frequencies. Associations that have been reported include smoking, air pollution, benzene, chemotherapy, parabens, polychlorinated biphenyl and scrotal heating, amongst others (Robbins et al. 2005, Tempest et al. 2005, 2008, Perry 2008, Xing et al. 2010, Ji et al. 2012, Mcauliffe et al. 2012, Figueroa et al. 2015, Governini et al. 2015, Radwan et al. 2016, Perry et al. 2016, Beal et al. 2017, Jurewicz et al. 2017, Rives et al. 2017, Zhang et al. 2018). It is important to note that such studies in humans are notoriously difficult to design, compare and reproduce, and it is extremely difficult to prove causation given the multitude of confounding factors that exist in such studies. Furthermore, the increases in sperm aneuploidy reported in these studies although significant, rarely reach the levels observed in infertile patients considered in subsequent sections. This holds true even in the case of highly cytotoxic chemotherapeutic drugs administered to cancer patients. These patients often present with significant but transient levels of sperm aneuploidy that may or may not reach the levels reported in infertile men (Tempest et al. 2008). Increasingly, spermatozoa are considered as potential biomarkers to assess reproductive toxicity, as invasive procedures are required to obtain other male or female germ cells (Macgregor et al. 1995, Anton \& Krawetz 2012). Studies involving rodent model systems have frequently shown that exposure to germinal mutagens can have significant consequences on reproductive outcomes including embryonic loss and increases in mutations and chromosomal abnormalities (Wyrobek 1993). Genotoxic and mutagenic studies frequently assess chromosome aberrations in somatic cells to determine whether compounds have carcinogenic potential through multiple techniques, including FISH, comet assay and the micronucleus assay, amongst others. Inclusion of sperm aneuploidy in such studies could also provide valuable information on reproductive toxicants and will ultimately provide a more comprehensive evaluation of carcinogenicity and reproductive toxicity (Mandrioli et al. 2016). In addition to investigating chromosome aneuploidy in sperm, other biomarkers should be considered to monitor exposure to environmental hazards, including sperm DNA integrity (DNA fragmentation), chromatin modifications (epigenetics) and nuclear organization amongst others (Anton \& Krawetz 2012). Emerging evidence suggests environmental factors can alter the establishment and maintenance of epigenetic modifications (Feil \& Fraga 2012). Such perturbations could have significant consequences on gene expression, health and disease and fertility (Zalensky \& Zalenskaya 2007, Hammoud et al. 2011, Aston et al. 2012, Millan et al. 2012, Hotaling \& Carrell 2014, loannou et al. 2017, loannou \& Tempest 2018). Assessment of the sperm epigenome is likely to play an increasingly important role in assessing reproductive toxicology and may be clinically relevant in the work-up of infertile men. Already commercially available tests are available to interrogate the sperm epigenome.

\section{Sperm aneuploidy levels in infertile men}

Virtually all published studies have reported higher frequencies of sperm aneuploidy in infertile males, compared to fertile or normozoospermic males (Shi \& Martin 2000, Tempest \& Griffin 2004, Templado et al. 2013), with the exception of a handful of studies (Miharu et al. 1994, Guttenbach et al. 1997). In general, similar aneuploidy frequencies are reported across studies. However, direct comparisons between studies can be challenging due to the heterogeneity of infertile patient phenotypes or controls used (proven fertile controls, normozoospermic patients, or both). Additionally, not all studies provide detailed semen parameter information. Differences also exist between studies in terms of the number of sperm scored, chromosomes analyzed, FISH probes utilized and scoring criteria used. The use of relatively standardized stringent scoring criteria attenuates the variability between studies considerably. However, not all studies provide detailed scoring criteria, numbers of raters or interrater variability (increased use of automated approaches will likely reduce many of these factors significantly). Additionally, studies often report levels of aneuploidy differently. For example, some may include or exclude nullisomy or diploidy, others may report total gonosomy aneuploidy, whereas others report $X X, X Y$ and $Y Y$ disomy individually. Sperm aneuploidy is also sometimes reported as a fold increase without providing raw numbers or percentages and different statistical methods are used to determine significance. Thus, direct comparisons between studies can be problematic, and these factors should be considered when looking 
at reported aneuploidy frequencies (Fig. 3). Despite these inherent challenges, the vast majority of studies reassuringly report comparable findings. The levels of sperm aneuploidy reported in different studies has been previously reviewed extensively (Shi \& Martin 2000, Tempest \& Griffin 2004, Templado et al. 2011, 2013). Overall, there is a two- to three-fold increase in sperm aneuploidy levels in infertile men compared to controls (Fig. 3). Yet, interindividual differences exist, with tenfold or higher frequencies reported in some infertile men (Tempest \& Griffin 2004, Harton \& Tempest 2012, Templado et al. 2013, Ioannou \& Tempest 2015). The existence of interindividual variation has been reported in fertile and infertile men and should be taken into account when considering reported global levels of sperm aneuploidy levels (Fig. 3). A correlation between the severity of semen parameters perturbations and the levels of sperm aneuploidy has been reported in the literature (Fig. 3). For example, NOA and severe oligoasthenoteratozoospermic (OAT) males often have significantly higher frequencies of sperm aneuploidy, compared to men with normal or milder semen parameter disturbances (Shi \& Martin 2000, Tempest \& Griffin 2004, Tempest et al. 2004). Data in NOA, and severe OAT, is often based on very low numbers due to insufficient sperm available for analysis. Notably, male partners of patients with recurrent pregnancy loss (RPL) and recurrent implantation failure (RIF) following ART are reported to have significantly higher levels of sperm aneuploidy (these patients will be considered in more detail in the subsequent section). Patients with numerical chromosome aberrations (e.g., 47,XXY and 47,XYY) and structural chromosome aberrations also exhibit very high levels of sperm aneuploidy for the chromosomes involved. Sperm aneuploidy levels range from 1 to $25 \%$ and 3 to $81 \%$ in individuals with numerical and structural chromosome aberrations, respectively (Fig. 3) (Harton \& Tempest 2012, loannou \& Tempest 2015). Since these studies often only test a handful of chromosomes (those involved in the aberration), sperm aneuploidy levels are likely to be even higher. Reported sperm aneuploidy levels in patients with numerical chromosome aberrations are high, but are lower than would be expected following meiotic segregation of three sex chromosomes (50\%), further supporting the existence of an unidentified male meiotic checkpoint. The huge variation in sperm aneuploidy frequencies in carriers of structural chromosome aberrations is likely to be affected by the type of aberration, size and location of the aberration and chromosome(s) involved. The large variation reported in these patients makes it extremely challenging to provide accurate risk assessments to patients regarding the risk of producing chromosomally unbalanced conceptions without sperm aneuploidy testing. Clearly, a patient with $3 \%$ aneuploid sperm would be counseled differently than a patient with $80 \%$ aneuploid sperm, for the tested chromosomes. Additionally, studies also report an

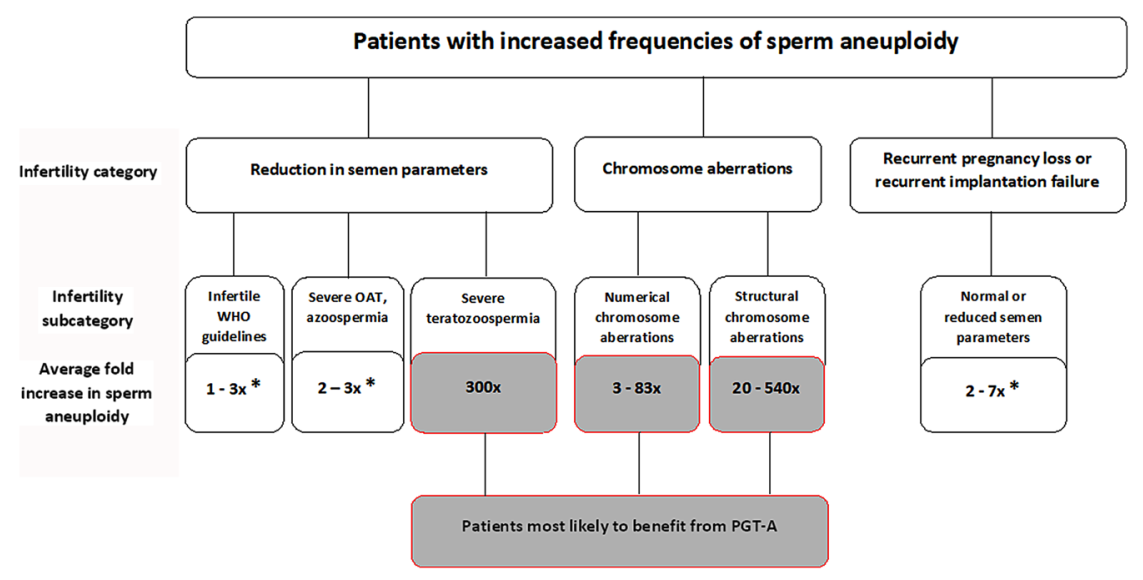

Figure 3 Overview of increased sperm aneuploidy levels in infertile patients and indication of which patient groups may benefit from preimplantation genetic testing for aneuploidy (PGT-A). Included in this figure are the major categories of infertile patients who are at risk of producing significantly higher levels of sperm aneuploidy compared to fertile or normozoospermic males (controls). Patients are split into three categories and six subcategories; for each subcategory, the average fold increase in sperm aneuploidy compiled from numerous studies is presented compared to control levels. The reduction in semen parameters group is split into three patient subcategories: mild semen parameter disturbances, severe oligoasthenoteratozoospermic (OAT), and rare severe teratozoospermic, as defined by the World Health Organization (WHO). Asterisks $\left(^{*}\right)$ represent patient groups who may potentially benefit from sperm aneuploidy screening, if high levels of sperm aneuploidy are found, these patients may benefit from genetic counseling and being offered PGT-A. The three subcategories with the highest levels of sperm aneuploidy include specific types of severe teratozoospermia, numerical and structural chromosome aberrations. Sperm aneuploidy screening in these groups can identify the patients with extremely high levels of aneuploid sperm. These patients should receive appropriate genetic counseling and be offered PGT-A so they can make informed decisions regarding the risks to their future offspring and to potentially improve their reproductive outcomes. Note: This figure should be interpreted with caution due to interindividual differences in sperm aneuploidy frequencies and challenges associated with cross study comparisons as outlined in the text. 
interchromosomal effect in patients, suggesting they may have a generalized increase in sperm aneuploidy for all chromosomes not just those involved in the chromosome aberration (Harton \& Tempest 2012). Some of the highest sperm aneuploidy frequencies have been reported in a handful of patients with severe teratozoospermia (e.g., semen samples containing high levels microcephalic and macrocephalic sperm), these rare patients have aneuploidy levels exceeding $50 \%$ for the few chromosomes investigated (Fig. 3) (Hotaling \& Carrell 2014).

\section{Sperm aneuploidy and recurrent pregnancy loss (RPL) and ART recurrent implantation failure (RIF)}

Loss of a clinically recognized pregnancy is relatively common occurring in $15-25 \%$ of pregnancies, however, a subset of pregnant women (less than 5\%) will experience RPL (Asrm 2012). RPL is defined by the American Society of Reproductive Medicine as two or more failed clinically recognized pregnancies. In about half of these cases, the underlying cause of RPL is idiopathic, and patient work-ups often focus on maternal factors (genetic, chromosomal, AMA, hormonal disturbances, uterine abnormalities, metabolic, infectious or immune disorders etc.) (ZidiJrah et al. 2016). Even less is currently understood about the underlying basis of RIF in some ART patients. Evaluation of the male partner in RPL and RIF is typically restricted to karyotype analysis and a basic semen parameter assessment. Karyotype analysis is important in both the male and female partner to rule out structural chromosome aberrations. Balanced carriers of structural aberrations are typically phenotypically normal, but they have a high risk of producing chromosomally unbalanced gametes following meiotic segregation of the structural aberration, which could result in RPL, RIF or congenital malformations (Stern et al. 1999). In recent years, the paternal contribution to RPL and RIF beyond semen parameters and karyotyping is being increasingly examined. Multiple studies suggest that increased levels of sperm aneuploidy may be a significant contributory factor in RPL and/or RIF (Rubio et al. 2001, Carrell et al. 2003, Bernardini et al. 2004, Collodel et al. 2009, Rubio et al. 2009, Neusser et al. 2015, Ramasamy et al. 2015, Zidi-Jrah et al. 2016, Esquerre-Lamare et al. 2018). These studies have reported a two- to seven-fold increase in sperm aneuploidy for the investigated chromosomes in these patients (Fig. 3) (Collodel et al. 2009, Kohn et al. 2016, Zidi-Jrah et al. 2016, Esquerre-Lamare et al. 2018). The majority of the aforementioned studies have focused on males with reduced semen parameters; however, a large study reported that up to $40 \%$ of male partners of RPL with normal semen parameters also had significantly increased sperm aneuploidy levels (Ramasamy et al. 2015). These findings suggest that the production of increased sperm aneuploidy in male patients with normal semen parameters may also contribute to RPL and RIF.

\section{Clinical consequences of increased sperm aneuploidy}

Infertile men with perturbations in their semen parameters frequently exhibit modest, but significant increases in sperm aneuploidy levels compared to their fertile/normozoospermic counterparts. The clinical relevance of increased levels of sperm aneuploidy in infertile males has not been fully elucidated. We and others argue that there is mounting evidence to suggest inclusion of sperm aneuploidy testing may be beneficial as part of the diagnostic work-up of select patients (Griffin et al. 2003, Gianaroli et al. 2005, Nagvenkar et al. 2005, Carrell 2008, Nicopoullos et al. 2008, Sanchez-Castro et al. 2009, Rodrigo et al. 2010, Harton \& Tempest 2012, Zidi-Jrah et al. 2016). Indirect evidence of a correlation between higher levels of chromosome aneuploidy and aneuploid offspring is provided by retrospective studies. These studies have identified high levels of sperm aneuploidy in the fathers of paternally derived aneuploid offspring (Blanco et al. 1998, Martinez-Pasarell et al. 1999, Moosani et al. 1999, Soares et al. 2001, Arnedo et al. 2006). Several studies have also reported correlations between high levels of sperm aneuploidy and recurrent intracytoplasmic sperm injection (ICSI) or ART failure (Burrello et al. 2003, Petit et al. 2005, Kahraman et al. 2006, Nicopoullos et al. 2008), increased levels of aneuploidy in preimplantation embryos (Gianaroli et al. 2005, Magli et al. 2009, Sanchez-Castro et al. 2009, Rodrigo et al. 2010, Coates et al. 2015) and lower pregnancy rates and live births (Nagvenkar et al. 2005). Moderate increases in sperm aneuploidy in infertile men have also been postulated to contribute to the higher levels of aneuploid offspring conceived after ICSI (Egozcue et al. 1997, Griffin et al. 2003). ICSI is the ART procedure used to treat severe male infertility and has enabled many of these patients to have biological children; ICSI was rapidly adopted in IVF clinics worldwide and is now often used in ART even in the absence of a male factor (Boulet et al. 2015). Despite the widespread use of ICSI, concerns have been raised regarding the genetic quality of the sperm used, and that it bypasses the 'fittest sperm' selection process present in natural conceptions or conventional IVF (Griffin et al. 2003). These concerns may be valid, as studies have reported an approximate three-fold increase in de novo chromosome aberrations in ICSI derived offspring, which mirrors the three-fold increase observed in sperm aneuploid frequencies in infertile men (Aboulghar et al. 2001, Bonduelle et al. 2002, Van Steirteghem et al. 2002, Devroey \& Van Steirteghem 2004). The findings of these studies suggest that aneuploid sperm are equally 
capable of fertilizing an oocyte than chromosomally normal sperm, which may lead to RIF, spontaneous abortions, RPL or viable aneuploid offspring.

\section{Why is sperm aneuploidy screening rarely considered in ART?}

Clearly, we can identify individuals who are at risk of producing high levels of sperm aneuploidy including those with: (1) structural or numerical chromosome aberrations; (2) azoospermia; (3) severeteratozoospermia; (4) severe OAT and (5) RPL and/or RIF. Then, why are these patients not routinely offered sperm aneuploidy screening as part of their diagnostic work-up? One issue is that ART providers may not be aware of the patients who are at an increased risk of producing higher levels of sperm aneuploidy and may benefit from screening. There are also technical challenges to consider that have perhaps limited the implementation of sperm aneuploidy screening. Typically only three to five chromosomes are analyzed by FISH. Thus, a genome-wide perspective of aneuploidy levels is not provided. Due to the relatively low levels of sperm aneuploidy, thousands of sperm are required to be scored, which is labor intensive. This issue is readily solvable with the advent of various automated systems that have the capability to accurately and reliably assess sperm aneuploidy (Netten et al. 1997, Baumgartner et al. 2001, Perry et al. 2007, 2011, Carrell \& Emery 2008, Molina et al. 2009, Tempest et al. 2010, Martinez et al. 2013, Branch et al. 2017). In reality, we are unable to test the individual sperm that will be used in ART; thus, it can only provide a generalized overview of the levels of aneuploidy in a semen sample. Perhaps one of the greatest challenges when considering the clinical relevance and utilization of sperm aneuploidy screening is the lack of a clearly established and validated sperm aneuploidy threshold (Kohn et al. 2016). These need to be developed by each individual laboratory performing sperm aneuploidy testing with large numbers of individuals. Critically, it also requires clinics to share ART outcome data with these labs in order to develop and assess clinical recommendations for sperm aneuploidy screening. With increasing sperm aneuploidy screening and ART outcome data, better correlations will be able to be made regarding the consequence of increased sperm aneuploidy, which will ultimately facilitate more accurate genetic counseling and risk assessments for patients at an increased risk of RIF, RPL, spontaneous abortions and aneuploid offspring. Often, when any type of genetic testing is undertaken, the result provides some quantifiable modified risk. Unfortunately, we are not there yet with sperm aneuploidy screening, which poses significant challenges for clinicians, genetic counselors and consequently patients. Several valid questions remain: (1) what is a clinically significant level of sperm aneuploidy, and (2) how does this translate to risk of chromosome aneuploidy in future offspring? Addressing these questions is complicated by the large maternal contribution to aneuploidy and the fact that PGT-A in embryos using SNP arrays have only reported paternally derived aneuploidies that arose post-zygotically (i.e., were mitotic rather than meiotic in origin) (Rabinowitz et al. 2012). However, the numbers of published embryos analyzed by SNP arrays remains relatively small especially given the large maternal contribution to chromosome aneuploidy. Thus, it is likely that the sample size may not yet be large enough to detect paternal trisomies that were meiotic in origin. Moreover, the PGT-A SNP array technology needs to be applied specifically in patient cohorts with an increased risk of sperm aneuploidy to determine whether this translates to an increase in paternally derived meiotic aneuploidies in preimplantation embryos.

Sperm aneuploidy is perhaps more straightforward to manage clinically in patients who exhibit very high levels of sperm aneuploidy, such as patients with constitutional structural or numerical chromosome aberrations or severe teratozoospermia. These patients should be counseled on the availability of PGT-A, prenatal genetic testing and the possibility of using donor sperm. The question remains, how should we clinically manage a two- to three-fold increase in sperm aneuploidy which is commonplace in infertile patients? This lack of clarity alongside the technical challenges discussed previously has precluded the widespread use of sperm aneuploidy testing as part of the work-up for those patients who might benefit. We also need to consider which chromosomes should be tested when assessing sperm aneuploidy frequencies clinically, given that typically only up to five chromosomes are tested. In most cases, the chromosomes tested focus on the clinically viable trisomies. Yet, these may not always be the most appropriate chromosomes to test. For example, in patients with structural or numerical chromosome aberrations, additional chromosomes not involved in the aberration should be also tested to identify any interchromosomal effect. Additionally, focusing on chromosomes that are not viable in an aneuploid state may be more informative in patients with RIF or RPL, as arrested embryonic development and recurrent spontaneous abortions are associated with this group. Given the potential clinical impact of sperm aneuploidy, we ought to also consider whether application of PGT-A may lead to better outcomes in patients with high levels of sperm aneuploidy undergoing ART.

\section{Preimplantation genetic testing for aneuploidy (PGT-A) in patients with increased sperm aneuploidy}

The principle behind the clinical application of PGT-A is that given the high levels of aneuploidy, the ability to identify euploid embryos will lead to increased rates 
of clinical pregnancies, reduced rates of miscarriages, reduced time to pregnancy and critically improved live birth rates following ART. Theoretically, PGT-A should not only improve outcomes but also reduce the financial burdens of multiple embryo transfers, emotional stress of miscarriages and alleviate the complications associated with multiple gestations through single embryo transfers. In practice, however, the PGT field has been fraught with challenges (Chen et al. 2018). Some of these challenges have been mitigated by changing the technology from FISH to array comparative genomic hybridization (aCGH), and more recently next generation sequencing. Additionally, many clinics have moved from day 3 blastomere biopsy to day 5 trophectoderm biopsy. However, these advances have also added more complexity to the field, including the detection of chromosome mosaicism and segmental aneuploidies, both of which appear to be common biological phenomena in the early preimplantation embryo (Fragouli et al. 2010, Babariya et al. 2017). Segmental aneuploidies can be inherited following the meiotic segregation of a balanced structural chromosome aberration resulting in unbalanced gametes, or they can arise due to a de novo error in meiosis or through a mitotic error during early gametogenesis (Vera-Rodriguez et al. 2016). De novo chromosome aberrations in natural conceptions as detected through amniocentesis are found at a low frequency (Warburton 1991), with segmental aneuploidies found in $\sim 6 \%$ of miscarriages (Martinez et al. 2010) and $~ 0.05 \%$ of liveborns (Wellesley et al. 2012). A huge variation in the incidence of segmental aneuploidies in preimplantation embryos has been reported, ranging from 5 to $70 \%$, with most studies reporting an incidence of around 10-15\% (Voullaire et al. 2000, Wells \& Delhanty 2000, Vanneste et al. 2009, Rabinowitz et al. 2012, Vera-Rodriguez et al. 2016, Babariya et al. 2017). These studies suggest that the majority of segmental chromosome aneuploidies detected by PGT are not compatible with fetal development and will likely spontaneously abort, fail to implant or if viable could be associated with congenital abnormalities in the affected offspring (Wellesley et al. 2012). It is noteworthy that segmental aneuploidies are not correlated with AMA and appear to be predominantly mitotic in origin. However, those that are meiotic in origin are twice as likely to be paternally, rather than maternally derived (Konstantinidis et al. 2016). This confirms some of the early human sperm hamster oocyte fusion karyotyping data that identified up to a six-fold increase in structural chromosome aberrations in sperm compared to oocytes (Martin 2008). This indicates that meiotic processes specific to males and/or the presence of DNA damage that is unable to be repaired within sperm may play a significant role in the origin of the majority of segmental aneuploidies (Babariya et al. 2017).
Following appropriate genetic counseling, some couples may wish to consider undertaking ART in conjunction with PGT-A, for indications other than AMA. The use of PGT-A in ART over the past decade has steadily increased in the United States especially for patients of AMA. Couples who are planning to undertake PGT-A with their ART cycle may not benefit from additional sperm aneuploidy testing. However, sperm aneuploidy testing may be useful in at risk patients to assist them in making an informed decision as to whether they wish to consider PGT-A, particularly in the absence of AMA. These at risk patients may not have previously considered, or been offered PGT-A (Fig. 3). Patients with the highest levels of sperm aneuploidy (e.g., structural or numerical chromosome aberrations, and severe teratozoospermia) will likely benefit the most from PGT-A. PGT-A may also be beneficial in patients with unexplained RPL or RIF with accompanying increased levels of sperm aneuploidy, and also perhaps those with sperm aneuploidy levels that are three-fold or higher compared to controls. Whether PGT-A may improve reproductive outcomes in these patients by reducing spontaneous abortions, time to pregnancy and aneuploid offspring and potentially increasing live birth rates remains inconclusive. Given the mounting evidence, this clearly warrants further investigation (Rubio et al. 2009, Murugappan et al. 2016, EsquerreLamare et al. 2018).

\section{Conclusions}

Male factors contribute to around $50 \%$ of infertility; nonetheless, up to $80 \%$ of male infertility remains unexplained. Furthermore, evidence is growing to suggest that sperm aneuploidy may contribute to infertility in select patients groups. Despite recent renewed interest in sperm aneuploidy, it remains to be seen whether assessment of sperm aneuploidy will become a more utilized clinical tool in the arsenal of infertility specialists. There are certainly patients who are at risk of having high levels of sperm aneuploidy, including those with structural and numerical chromosome aberrations, azoospermia, severe OAT and teratozoospermia and those who have experienced unexplained RPL and RIF. Numerous studies have suggested that even a modest (two- to three-fold) increase in sperm aneuploidy seemingly translates to a similar increased frequency of aneuploid embryos, ICSI failure, RIF, RPL and aneuploid offspring. Thus, these patients would probably benefit from sperm aneuploidy screening with appropriate genetic counseling that involved discussion of risk to future offspring, availability of PGT-A, appropriate prenatal screening and testing, and the option of donor sperm in rare cases of very high levels of sperm aneuploidy. Recent technological advances allow us to directly examine the 
paternal contribution to meiotic aneuploidies through PGT-A testing (e.g., SNP arrays). Studies are needed to address the parental contribution to aneuploidy and should also focus on the aforementioned male patients at a higher risk of producing aneuploid sperm. If these studies reveal a clinically relevant paternal meiotic contribution to segmental and whole chromosome aneuploidy, routine clinical implementation of sperm aneuploidy should follow, at least for those patients at an increased risk. It seems likely that increased access to reliable and accurate automated sperm aneuploidy scoring systems should reduce costs and technical challenges currently associated with sperm aneuploidy screening, which may increase clinical uptake. Future developments may leverage existing technology to further facilitate the rapid detection of more chromosomes simultaneously in a single cell. Examples include the possible development of novel fluorescent dyes to replace traditional organic fluorochromes, such as quantum dots, which has already been applied to FISH with mixed success (loannou et al. 2009, Yusuf et al. 2011, Hwang et al. 2015, Wang et al. 2018). Quantum dots are inorganic fluorochromes that are resistant to photobleaching and excite at a wide range of wavelengths but emit light in a very narrow band (controlled by particle size) (loannou et al. 2009). Thus, they have improved photostability and a greater potential for multiplexing experiments compared to traditional organic fluorochromes. Alternative rapid automated systems such as FISH-flow cytometry has emerged for some FISH applications and may be applicable to sperm aneuploidy, and could be potentially combined with quantum dots (Kapoor et al. 2009, Arrigucci et al. 2017). Quantum dots and FISH-flow cytometry have been around for many years but have not been rapidly adopted in the field of cytogenetics. It remains to be seen if these will be applicable to sperm aneuploidy testing given the unique characteristics of the sperm cell. Another interesting area of future development lies in microfluidics, which is increasingly being applied to various fields of medicine and has been proposed as an additional tool to sort and analyze sperm for ART (Nosrati et al. 2017, Quinn et al. 2018, Samuel et al. 2018). It is plausible that such microfluidic devices may be able to preferentially identify and select certain sperm characteristics and even possibly reduce the chance of selecting aneuploid sperm for use in ART, although this remains to be tested. Perhaps the most transformative developments lie with the rapid advancements in genomics technology, which may minimize the current technical limitations of sperm aneuploidy screening by FISH. For example, microarray technology is now available in microtiter plate formats. This facilitates high volume, low cost genome-wide analysis, and could potentially be used not only to evaluate whole chromosome aneuploidy in sperm but also segmental aneuploidy, which has a relatively high paternal contribution. The increased uptake in clinical genome sequencing, gene expression assays and epigenetic tests may eventually lead to additional clinically useful and diagnostic test(s) for male infertility. This will likely lead to a better understanding of the paternal contribution to early embryogenesis, and reveal the underlying genetic basis of unexplained male infertility. This will hopefully lead to more appropriate use of ART with better outcomes, and potentially the development of alternative therapies and lifestyle interventions that may reduce current reliance on ART for treatment. In the near future, it seems likely that genome-wide highthroughput technologies will also be utilized to assess the epigenetic potential of sperm, identify rare genomic variants, CNVs and sperm aneuploidy.

Despite the need to develop new tests and approaches for male factor infertility, the field must exercise caution before it adopts new tests. Any new test or therapeutic intervention needs to be carefully validated with close attention paid to patient phenotypes. The large degree of heterogeneity in male infertility phenotypes is often overlooked, but this is critical to determine which patients may benefit from new tests and therapeutic interventions, and which will not. The successful clinical implementation of any tests will also require a shift in attitude amongst many clinicians and embryologists, away from 'you just need any sperm', to a realization that the paternal genome plays a bigger contribution to early embryogenesis and IVF outcomes than is currently appreciated by many. Assessment of sperm aneuploidy may ultimately lead to improved ART outcomes in select patients. It is clear that research has awoken the silent vessel of the sperm cell; we now know it delivers an epigenetically primed genome that is likely crucial for early embryogenesis. Historically, the data from prenatal studies, products of conception and live births suggest that the paternal contribution to aneuploidy is small relative to the maternal contribution. However, many of these studies were performed before the advent of ICSI, which has enabled many infertile men to have biological children. Current evidence suggests that the paternal contribution to aneuploidy in infertile men may be considerably higher than we currently perceive. Furthermore, meiotic segmental aneuploidies in preimplantation embryos are more frequently paternal in origin, which further increases the paternal contribution to aneuploidy. The time is now to carefully reevaluate the paternal contribution to human aneuploidy in infertile patients through well designed research and clinical studies.

\section{Declaration of interest}

The authors declare that there is no conflict of interest that could be perceived as prejudicing the impartiality of this review. 


\section{Funding}

This research did not receive any specific grant from any funding agency in the public, commercial or not-for-profit sector.

\section{Acknowledgements}

The authors would like to thank Renee H Martin for providing the images of meiotic spermatocytes that were used to produce Fig. 2.

\section{References}

Aboulghar H, Aboulghar M, Mansour R, Serour G, Amin Y \& Al-Inany H 2001 A prospective controlled study of karyotyping for 430 consecutive babies conceived through intracytoplasmic sperm injection. Fertility and Sterility 76 249-253. (https://doi.org/10.1016/S0015-0282(01)01927-6)

Andreescu NI, Cosma M, Farcas SS, Stoian M, Amzar DG \& Puiu M 2016 Assessment of chromosomal aneuploidies in sperm of infertile males by using FISH technique. Romanian Journal of Morphology and Embryology 57 173-178.

Anton E \& Krawetz SA 2012 Spermatozoa as biomarkers for the assessment of human male infertility and genotoxicity. Systems Biology in Reproductive Medicine 58 41-50. (https://doi.org/10.3109/1939636 8.2011.637152)

Arnedo N, Templado C, Sanchez-Blanque Y, Rajmil O \& Nogues C 2006 Sperm aneuploidy in fathers of Klinefelter's syndrome offspring assessed by multicolour fluorescent in situ hybridization using probes for chromosomes 6, 13, 18, 21, 22, X and Y. Human Reproduction 21 524-528. (https://doi.org/10.1093/humrep/dei321)

Arrigucci R, Bushkin Y, Radford F, Lakehal K, Vir P, Pine R, Martin D, Sugarman J, Zhao Y, Yap GS et al. 2017 FISH-flow, a protocol for the concurrent detection of mRNA and protein in single cells using fluorescence in situ hybridization and flow cytometry. Nature Protocols 12 1245-1260. (https://doi.org/10.1038/nprot.2017.039)

ASRM 2012 Evaluation and treatment of recurrent pregnancy loss: a committee opinion. Fertility and Sterility 98 1103-1111. (https://doi. org/10.1016/j.fertnstert.2012.06.048)

Aston KI, Punj V, Liu L \& Carrell DT 2012 Genome-wide sperm deoxyribonucleic acid methylation is altered in some men with abnormal chromatin packaging or poor in vitro fertilization embryogenesis. Fertility and Sterility 97 285-292. (https://doi.org/10.1016/j. fertnstert.2011.11.008)

Babariya D, Fragouli E, Alfarawati S, Spath K \& Wells D 2017 The incidence and origin of segmental aneuploidy in human oocytes and preimplantation embryos. Human Reproduction 32 2549-2560. (https:// doi.org/10.1093/humrep/dex324)

Baumgartner A, Schmid TE, Maerz HK, Adler ID, Tarnok A \& Nuesse M 2001 Automated evaluation of frequencies of aneuploid sperm by laser-scanning cytometry (LSC). Cytometry 44 156-160. (https://doi.org/10.1002/1097-0320(20010601)44:2<156::AIDCYTO1096>3.0.CO;2-5)

Beal MA, Yauk CL \& Marchetti F 2017 From sperm to offspring: assessing the heritable genetic consequences of paternal smoking and potential public health impacts. Mutation Research 773 26-50. (https://doi. org/10.1016/j.mrrev.2017.04.001)

Bernardini LM, Costa M, Bottazzi C, Gianaroli L, Magli MC, Venturini PL, Francioso R, Conte N \& Ragni N 2004 Sperm aneuploidy and recurrent pregnancy loss. Reproductive BioMedicine Online 9 312-320. (https:// doi.org/10.1016/S1472-6483(10)62147-5)

Blanco J, Gabau E, Gomez D, Baena N, Guitart M, Egozcue J \& Vidal F 1998 Chromosome 21 disomy in the spermatozoa of the fathers of children with trisomy 21 , in a population with a high prevalence of down syndrome: increased incidence in cases of paternal origin. American Journal of Human Genetics 63 1067-1072. (https://doi. org/10.1086/302058)

Bonduelle M, Van Assche E, Joris H, Keymolen K, Devroey P, Van Steirteghem A \& Liebaers I 2002 Prenatal testing in ICSI pregnancies: incidence of chromosomal anomalies in 1586 karyotypes and relation to sperm parameters. Human Reproduction 17 2600-2614. (https://doi. org/10.1093/humrep/17.10.2600)

Boulet SL, Mehta A, Kissin DM, Warner L, Kawwass JF \& Jamieson DJ 2015 Trends in use of and reproductive outcomes associated with intracytoplasmic sperm injection. JAMA 313 255-263. (https://doi. org/10.1001/jama.2014.17985)

Boyle S, Gilchrist S, Bridger JM, Mahy NL, Ellis JA \& Bickmore WA 2001 The spatial organization of human chromosomes within the nuclei of normal and emerin-mutant cells. Human Molecular Genetics 10 211-219. (https://doi.org/10.1093/hmg/10.3.211)

Branch F, Nguyen G, Porter N, Young HA, Martenies SE, Mccray N, Deloid G, Popratiloff A \& Perry MJ 2017 Semi-automated scoring of triple-probe FISH in human sperm using confocal microscopy. Cytometry A 91 859-866. (https://doi.org/10.1002/cyto.a.23126)

Burrello N, Vicari E, Shin P, Agarwal A, De Palma A, Grazioso C, D'agata R \& Calogero AE 2003 Lower sperm aneuploidy frequency is associated with high pregnancy rates in ICSI programmes. Human Reproduction $\mathbf{1 8}$ 1371-1376. (https://doi.org/10.1093/humrep/deg299)

Carrell DT 2008 The clinical implementation of sperm chromosome aneuploidy testing: pitfalls and promises. Journal of Andrology 29 124-133. (https://doi.org/10.2164/jandrol.107.003699)

Carrell DT 2013 The reproductive fitness of the human male gamete. In: Paternal Influences on Human Reproductive Success, pp 1-5. Ed DT Carrell. Cambridge University Press, Cambridge, UK.

Carrell DT \& Emery BR 2008 Use of automated imaging and analysis technology for the detection of aneuploidy in human sperm. Fertility and Sterility 90 434-437. (https://doi.org/10.1016/j. fertnstert.2007.06.095)

Carrell DT, Wilcox AL, Lowy L, Peterson CM, Jones KP, Erickson L, Campbell B, Branch DW \& Hatasaka HH 2003 Elevated sperm chromosome aneuploidy and apoptosis in patients with unexplained recurrent pregnancy loss. Obstetrics and Gynecology 101 1229-1235.

Chandra A, Martinez GM, Mosher WD, Abma JC \& Jones J 2005 Fertility, family planning, and reproductive health of U.S. women: data from the 2002 National Survey of Family Growth. Vital and Health Statistics 23 $1-160$.

Chen HF, Chen SU, Ma GC, Hsieh ST, Tsai HD, Yang YS \& Chen M 2018 Preimplantation genetic diagnosis and screening: current status and future challenges. Journal of the Formosan Medical Association 117 94-100. (https://doi.org/10.1016/j.jfma.2017.08.006)

Coates A, Hesla JS, Hurliman A, Coate B, Holmes E, Matthews R, Mounts EL, Turner KJ, Thornhill AR \& Griffin DK 2015 Use of suboptimal sperm increases the risk of aneuploidy of the sex chromosomes in preimplantation blastocyst embryos. Fertility and Sterility 104 866-872. (https://doi.org/10.1016/j.fertnstert.2015.06.033)

Collodel G, Giannerini V, Antonio Pascarelli N, Federico MG, Comodo F \& Moretti E 2009 TEM and FISH studies in sperm from men of couples with recurrent pregnancy loss. Andrologia 41 352-360. (https://doi. org/10.1111/j.1439-0272.2009.00936.x)

De Kretser DM 1997 Male infertility. Lancet 349 787-790. (https://doi. org/10.1016/S0140-6736(96)08341-9)

Devroey P \& Van Steirteghem A 2004 A review of ten years experience of ICSI. Human Reproduction Update 10 19-28. (https://doi.org/10.1093/ humupd/dmh004)

Donate A, Estop AM, Giraldo J \& Templado C 2016 Paternal age and numerical chromosome abnormalities in human spermatozoa. Cytogenetic and Genome Research 148 241-248. (https://doi. org/10.1159/000446724)

Egozcue J, Blanco J \& Vidal F 1997 Chromosome studies in human sperm nuclei using fluorescence in-situ hybridization (FISH). Human Reproduction Update 3 441-452. (https://doi.org/10.1093/ humupd/3.5.441)

Esquerre-Lamare C, Walschaerts $M$, Chansel Debordeaux L, Moreau J, Bretelle F, Isus F, Karsenty G, Monteil L, Perrin J, Papaxanthos-Roche A et al. 2018 Sperm aneuploidy and DNA fragmentation in unexplained recurrent pregnancy loss: a multicenter case-control study. Basic and Clinical Andrology 28 4. (https://doi.org/10.1186/s12610-018-0070-6)

Feil R \& Fraga MF 2012 Epigenetics and the environment: emerging patterns and implications. Nature Reviews Genetics 13 97-109. (https:// doi.org/10.1038/nrg3142)

Ferguson KA, Wong EC, Chow V, Nigro M \& Ma S 2007 Abnormal meiotic recombination in infertile men and its association with sperm 
aneuploidy. Human Molecular Genetics 16 2870-2879. (https://doi. org/10.1093/hmg/ddm246)

Ferlin A, Garolla A \& Foresta C 2005 Chromosome abnormalities in sperm of individuals with constitutional sex chromosomal abnormalities. Cytogenetic and Genome Research 111 310-316. (https://doi. org/10.1159/000086905)

Figueroa ZI, Young HA, Meeker JD, Martenies SE, Barr DB, Gray G \& Perry MJ 2015 Dialkyl phosphate urinary metabolites and chromosomal abnormalities in human sperm. Environmental Research 143 256-265. (https://doi.org/10.1016/j.envres.2015.10.021)

Fonseka KG \& Griffin DK 2011 Is there a paternal age effect for aneuploidy? Cytogenetic and Genome Research 133 280-291. (https:// doi.org/10.1159/000322816)

Fragouli E, Katz-Jaffe M, Alfarawati S, Stevens J, Colls P, Goodall NN, Tormasi S, Gutierrez-Mateo C, Prates R, Schoolcraft WB et al. 2010 Comprehensive chromosome screening of polar bodies and blastocysts from couples experiencing repeated implantation failure. Fertility and Sterility 94 875-887. (https://doi.org/10.1016/j.fertnstert.2009.04.053)

Garcia-Cruz R, Casanovas A, Brieno-Enriquez M, Robles P, Roig I, Pujol A, Cabero L, Durban M \& Garcia Caldes M 2010 Cytogenetic analyses of human oocytes provide new data on non-disjunction mechanisms and the origin of trisomy 16. Human Reproduction 25 179-191. (https://doi. org/10.1093/humrep/dep347)

Garcia-Ferreyra J, Hilario R \& Duenas J 2018 High percentages of embryos with 21, 18 or 13 trisomy are related to advanced paternal age in donor egg cycles. JBRA Assisted Reproduction 22 26-34. (https://doi. org/10.5935/1518-0557.20180004)

Gianaroli L, Magli MC \& Ferraretti AP 2005 Sperm and blastomere aneuploidy detection in reproductive genetics and medicine. Journal of Histochemistry and Cytochemistry 53 261-267. (https://doi. org/10.1369/jhc.4B6434.2005)

Gonsalves J, Sun F, Schlegel PN, Turek PJ, Hopps CV, Greene C, Martin RH \& Pera RA 2004 Defective recombination in infertile men. Human Molecular Genetics 13 2875-2883. (https://doi.org/10.1093/hmg/ddh302)

Governini L, Guerranti C, De Leo V, Boschi L, Luddi A, Gori M, Orvieto R \& Piomboni P 2015 Chromosomal aneuploidies and DNA fragmentation of human spermatozoa from patients exposed to perfluorinated compounds. Andrologia 47 1012-1019. (https://doi.org/10.1111/ and.12371)

Griffin DK, Abruzzo MA, Millie EA, Sheean LA, Feingold E, Sherman SL \& Hassold TJ 1995 Non-disjunction in human sperm: evidence for an effect of increasing paternal age. Human Molecular Genetics 4 2227-2232. (https://doi.org/10.1093/hmg/4.12.2227)

Griffin DK, Hyland P, Tempest HG \& Homa ST 2003 Safety issues in assisted reproduction technology: should men undergoing ICSI be screened for chromosome abnormalities in their sperm? Human Reproduction 18 229-235. (https://doi.org/10.1093/humrep/deg044)

Gruhn JR, Rubio C, Broman KW, Hunt PA \& Hassold T 2013 Cytological studies of human meiosis: sex-specific differences in recombination originate at, or prior to, establishment of double-strand breaks. PLoS ONE 8 e85075. (https://doi.org/10.1371/journal.pone.0085075)

Guttenbach M, Martinez-Exposito MJ, Michelmann HW, Engel W \& Schmid M 1997 Incidence of diploid and disomic sperm nuclei in 45 infertile men. Human Reproduction 12 468-473. (https://doi. org/10.1093/humrep/12.3.468)

Hammoud SS, Nix DA, Hammoud AO, Gibson M, Cairns BR \& Carrell DT 2011 Genome-wide analysis identifies changes in histone retention and epigenetic modifications at developmental and imprinted gene loci in the sperm of infertile men. Human Reproduction 26 2558-2569. (https://doi.org/10.1093/humrep/der192)

Harton GL \& Tempest HG 2012 Chromosomal disorders and male infertility. Asian Journal of Andrology 14 32-39. (https://doi.org/10.1038/ aja.2011.66)

Hassold T, Hunt PA \& Sherman S 1993 Trisomy in humans: incidence, origin and etiology. Current Opinion in Genetics and Development 3 398-403. (https://doi.org/10.1016/0959-437X(93)90111-2)

Hassold T, Sherman S \& Hunt P 2000 Counting cross-overs: characterizing meiotic recombination in mammals. Human Molecular Genetics 9 2409-2419. (https://doi.org/10.1093/hmg/9.16.2409)

Hotaling J \& Carrell DT 2014 Clinical genetic testing for male factor infertility: current applications and future directions. Andrology 2 339-350. (https://doi.org/10.1111/j.2047-2927.2014.00200.x)
Hwang G, Lee H \& Lee J 2015 Direct fluorescence in situ hybridization on human metaphase chromosomes using quantum dot-platinum labeled DNA probes. Biochemical and Biophysical Research Communications 467 328-333. (https://doi.org/10.1016/j.bbrc.2015.09.161)

loannou D \& Tempest HG 2015 Meiotic nondisjunction: insights into the origin and significance of aneuploidy in human spermatozoa. Advances in Experimental Medicine and Biology 868 1-21. (https://doi. org/10.1007/978-3-319-18881-2_1)

loannou D \& Tempest HG 2018 Does genome organization matter in spermatozoa? A refined hypothesis to awaken the silent vessel. Systems Biology in Reproductive Medicine 64518-534. (https://doi.org/10.1080/ 19396368.2017.1421278)

Ioannou D, Tempest HG, Skinner BM, Thornhill AR, Ellis M \& Griffin DK 2009 Quantum dots as new-generation fluorochromes for FISH: an appraisal. Chromosome Research 17 519-530. (https://doi.org/10.1007/ s10577-009-9051-0)

Ioannou D, Miller D, Griffin DK \& Tempest HG 2016 Impact of sperm DNA chromatin in the clinic. Journal of Assisted Reproduction and Genetics 33 157-166. (https://doi.org/10.1007/s10815-015-0624-x)

Ioannou D, Millan NM, Jordan E \& Tempest HG 2017 A new model of sperm nuclear architecture following assessment of the organization of centromeres and telomeres in three-dimensions. Scientific Reports 7 41585. (https://doi.org/10.1038/srep41585)

Ji Z, Weldon RH, Marchetti F, Chen H, Li G, Xing C, Kurtovich E, Young S, Schmid TE, Waidyanatha S et al. 2012 Comparison of aneuploidies of chromosomes 21, $X$, and $Y$ in the blood lymphocytes and sperm of workers exposed to benzene. Environmental and Molecular Mutagenesis 53 218-226. (https://doi.org/10.1002/em.21683)

Jurewicz J, Radwan M, Wielgomas B, Klimowska A, Kaluzny P, Radwan P, Jakubowski L \& Hanke W 2017 Environmental exposure to parabens and sperm chromosome disomy. International Journal of Environmental Health Research 27 332-343. (https://doi.org/10.1080/09603123.2017 .1339784)

Kahraman S, Findikli N, Biricik A, Oncu N, Ogur C, Sertyel S, Karlikaya G, Karagozoglu H \& Saglam Y 2006 Preliminary FISH studies on spermatozoa and embryos in patients with variable degrees of teratozoospermia and a history of poor prognosis. Reproductive BioMedicine Online 12 752-761. (https://doi.org/10.1016/S1472-6483(10)61087-5)

Kapoor V, Hakim FT, Rehman N, Gress RE \& Telford WG 2009 Quantum dots thermal stability improves simultaneous phenotype-specific telomere length measurement by FISH-flow cytometry. Journal of Immunological Methods 344 6-14. (https://doi.org/10.1016/j. jim.2009.02.004)

Kavitha P \& Malini SS 2014 Positive association of sperm dysfunction in the pathogenesis of recurrent pregnancy loss. Journal of Clinical and Diagnostic Research 8 OC07-OC10. (https://doi.org/10.7860/ JCDR/2014/9109.5172)

Kohn TP, Kohn JR, Darilek S, Ramasamy R \& Lipshultz L 2016 Genetic counseling for men with recurrent pregnancy loss or recurrent implantation failure due to abnormal sperm chromosomal aneuploidy. Journal of Assisted Reproduction and Genetics 33 571-576. (https://doi. org/10.1007/s10815-016-0702-8)

Konstantinidis M, Milligan K, Berkeley AS, Kennedy J, Maxson W, Racosky C, Wells D \& Munne S 2016 Use of a single nucleotide polymorphism (SNP) arrays and next generation sequencing (NGS) to study the incidence, type and origin of aneuploidy in the human preimplantation embryo. Fertility and Sterility 106 e22-e23. (https://doi. org/10.1016/j.fertnstert.2016.07.076)

Lewis SE 2007 Is sperm evaluation useful in predicting human fertility? Reproduction 134 31-40. (https://doi.org/10.1530/REP-07-0152)

Lu S, Zong C, Fan W, Yang M, Li J, Chapman AR, Zhu P, Hu X, Xu L, Yan L et al. 2012 Probing meiotic recombination and aneuploidy of single sperm cells by whole-genome sequencing. Science 338 1627-1630. (https://doi.org/10.1126/science.1229112)

Macgregor JT, Tucker JD, Eastmond DA \& Wyrobek AJ 1995 Integration of cytogenetic assays with toxicology studies. Environmental and Molecular Mutagenesis $25 \quad 328-337 . \quad$ (https://doi.org/10.1002/ em.2850250411)

Magli MC, Gianaroli L, Ferraretti AP, Gordts S, Fredericks V \& Crippa A 2009 Paternal contribution to aneuploidy in preimplantation embryos. Reproductive BioMedicine Online 18 536-542. (https://doi.org/10.1016/ S1472-6483(10)60131-9) 
Mandrioli D, Belpoggi F, Silbergeld EK \& Perry MJ 2016 Aneuploidy: a common and early evidence-based biomarker for carcinogens and reproductive toxicants. Environmental Health 15 97. (https://doi. org/10.1186/s12940-016-0180-6)

Martin RH 2006 Meiotic chromosome abnormalities in human spermatogenesis. Reproductive Toxicology 22 142-147. (https://doi. org/10.1016/j.reprotox.2006.03.013)

Martin RH 2008 Meiotic errors in human oogenesis and spermatogenesis. Reproductive BioMedicine Online 16 523-531. (https://doi.org/10.1016/ S1472-6483(10)60459-2)

Martinez MC, Mendez C, Ferro J, Nicolas M, Serra V \& Landeras J 2010 Cytogenetic analysis of early nonviable pregnancies after assisted reproduction treatment. Fertility and Sterility 93 289-292. (https://doi. org/10.1016/j.fertnstert.2009.07.989)

Martinez G, Gillois P, Le Mitouard M, Borye R, Esquerre-Lamare C, Satre V, Bujan L \& Hennebicq S 2013 FISH and tips: a large scale analysis of automated versus manual scoring for sperm aneuploidy detection. Basic and Clinical Andrology 23 13. (https://doi.org/10.1186/2051-4190-23-13)

Martinez-Pasarell O, Nogues C, Bosch M, Egozcue J \& Templado C 1999 Analysis of sex chromosome aneuploidy in sperm from fathers of Turner syndrome patients. Human Genetics 104 345-349. (https://doi. org/10.1007/s004390050964)

Mcauliffe ME, Williams PL, Korrick SA, Altshul LM \& Perry MJ 2012 Environmental exposure to polychlorinated biphenyls and $\mathrm{p}, \mathrm{p}^{\prime}$-DDE and sperm sex-chromosome disomy. Environmental Health Perspectives 120 535-540. (https://doi.org/10.1289/ehp.1104017)

Mclay DW \& Clarke HJ 2003 Remodelling the paternal chromatin at fertilization in mammals. Reproduction 125 625-633. (https://doi. org/10.1530/rep.0.1250625)

Miharu N, Best RG \& Young SR 1994 Numerical chromosome abnormalities in spermatozoa of fertile and infertile men detected by fluorescence in situ hybridization. Human Genetics 93 502-506.

Millan NM, Lau P, Hann M, loannou D, Hoffman D, Barrionuevo $M$, Maxson W, Ory S \& Tempest HG 2012 Hierarchical radial and polar organisation of chromosomes in human sperm. Chromosome Research 20 875-887. (https://doi.org/10.1007/s10577-012-9323-y)

Molina O, Sarrate Z, Vidal F \& Blanco J 2009 FISH on sperm: spot-counting to stop counting? Not yet. Fertility and Sterility 92 1474-1480. (https:// doi.org/10.1016/j.fertnstert.2008.07.1779)

Moosani N, Chernos J, Lowry RB, Martin RH \& Rademaker A 1999 A $47, X X Y$ fetus resulting from ICSI in a man with an elevated frequency of 24,XY spermatozoa. Human Reproduction 14 1137-1138. (https://doi. org/10.1093/humrep/14.4.1137)

Murugappan G, Shahine LK, Perfetto CO, Hickok LR \& Lathi RB 2016 Intent to treat analysis of in vitro fertilization and preimplantation genetic screening versus expectant management in patients with recurrent pregnancy loss. Human Reproduction 31 1668-1674. (https:// doi.org/10.1093/humrep/dew135)

Nagaoka SI, Hassold TJ \& Hunt PA 2012 Human aneuploidy: mechanisms and new insights into an age-old problem. Nature Reviews Genetics 13 493-504. (https://doi.org/10.1038/nrg3245)

Nagvenkar P, Zaveri K \& Hinduja I 2005 Comparison of the sperm aneuploidy rate in severe oligozoospermic and oligozoospermic men and its relation to intracytoplasmic sperm injection outcome. Fertility and Sterility 84 925-931. (https://doi.org/10.1016/j.fertnstert.2005.04.048)

Netten H, Young IT, Van Vliet LJ, Tanke HJ, Vroljik H \& Sloos WC 1997 FISH and chips: automation of fluorescent dot counting in interphase cell nuclei. Cytometry 28 1-10. (https://doi.org/10.1002/(SICl)10970320(19970501)28:1<1::AID-CYTO1>3.0.CO;2-K)

Neusser M, Rogenhofer N, Durl S, Ochsenkuhn R, Trottmann M, Jurinovic V, Steinlein O, Von Schonfeldt V, Muller S \& Thaler CJ 2015 Increased chromosome 16 disomy rates in human spermatozoa and recurrent spontaneous abortions. Fertility and Sterility $1041130 . e 1131-$ 1137.e1110. (https://doi.org/10.1016/j.fertnstert.2015.07.1160)

Nicopoullos JD, Gilling-Smith C, Almeida PA, Homa S, Nice L, Tempest H \& Ramsay JW 2008 The role of sperm aneuploidy as a predictor of the success of intracytoplasmic sperm injection? Human Reproduction 23 240-250. (https://doi.org/10.1093/humrep/dem395)

Nosrati R, Graham PJ, Zhang B, Riordon J, Lagunov A, Hannam TG, Escobedo C, Jarvi K \& Sinton D 2017 Microfluidics for sperm analysis and selection. Nature Reviews Urology 14 707-730. (https://doi. org/10.1038/nrurol.2017.175)
Pang MG, Hoegerman SF, Cuticchia AJ, Moon SY, Doncel GF, Acosta AA \& Kearns WG 1999 Detection of aneuploidy for chromosomes 4, 6, 7, 8, 9, $10,11,12,13,17,18,21, \mathrm{X}$ and $\mathrm{Y}$ by fluorescence in-situ hybridization in spermatozoa from nine patients with oligoasthenoteratozoospermia undergoing intracytoplasmic sperm injection. Human Reproduction $\mathbf{1 4}$ 1266-1273. (https://doi.org/10.1093/humrep/14.5.1266)

Perry MJ 2008 Effects of environmental and occupational pesticide exposure on human sperm: a systematic review. Human Reproduction Update 14 233-242. (https://doi.org/10.1093/humupd/dmm039)

Perry MJ, Chen X \& Lu X 2007 Automated scoring of multiprobe FISH in human spermatozoa. Cytometry A 71 968-972. (https://doi.org/10.1002/ cyto.a.20468)

Perry MJ, Chen X, Mcauliffe ME, Maity A \& Deloid GM 2011 Semiautomated scoring of triple-probe FISH in human sperm: methods and further validation. Cytometry A 79 661-666. (https://doi.org/10.1002/ cyto.a.21078)

Perry MJ, Young HA, Grandjean P, Halling J, Petersen MS, Martenies SE, Karimi P \& Weihe P 2016 Sperm aneuploidy in faroese men with lifetime exposure to dichlorodiphenyldichloroethylene (p,p-DDE) and polychlorinated biphenyl (PCB) pollutants. Environmental Health Perspectives 124 951-956. (https://doi.org/10.1289/ehp.1509779)

Petit FM, Frydman N, Benkhalifa M, Le Du A, Aboura A, Fanchin R, Frydman R \& Tachdjian G 2005 Could sperm aneuploidy rate determination be used as a predictive test before intracytoplasmic sperm injection? Journal of Andrology 26 235-241. (https://doi. org/10.1002/j.1939-4640.2005.tb01090.x)

Quinn MM, Jalalian L, Ribeiro S, Ona K, Demirci U, Cedars MI \& Rosen MP 2018 Microfluidic sorting selects sperm for clinical use with reduced DNA damage compared to density gradient centrifugation with swim-up in split semen samples. Human Reproduction 33 1388-1393. (https://doi.org/10.1093/humrep/dey239)

Rabinowitz M, Ryan A, Gemelos G, Hill M, Baner J, Cinnioglu C, Banjevic M, Potter D, Petrov DA \& Demko Z 2012 Origins and rates of aneuploidy in human blastomeres. Fertility and Sterility 97 395-401. (https://doi.org/10.1016/j.fertnstert.2011.11.034)

Rademaker A, Spriggs E, Ko E \& Martin R 1997 Reliability of estimates of diploid human spermatozoa using multicolour fluorescence in-situ hybridization. Human Reproduction 12 77-79.

Radwan M, Jurewicz J, Sobala W, Brze Nicki S, Radwan P, Jakubowski L, Hawula W, Ulanska A \& Hanke W 2016 Human sperm aneuploidy after exposure to polycyclic aromatic hydrocarbons. Reproduction, Fertility and Development 28 1376-1381. (https://doi.org/10.1071/RD14063)

Ramasamy R, Scovell JM, Kovac JR, Cook PJ, Lamb DJ \& Lipshultz LI 2015 Fluorescence in situ hybridization detects increased sperm aneuploidy in men with recurrent pregnancy loss. Fertility and Sterility 103906. e901-909.e901. (https://doi.org/10.1016/j.fertnstert.2015.01.029)

Rives N, Walschaerts M, Setif V, Hennebicq S, Saias J, Brugnon F, Auger J, Berthaut I, Szerman E, Daudin M et al. 2017 Sperm aneuploidy after testicular cancer treatment: data from a prospective multicenter study performed within the French Centre d'Etude et de Conservation des Oeufs et du Sperme network. Fertility and Sterility 107 580.e581-588. e581. (https://doi.org/10.1016/j.fertnstert.2016.11.015)

Robbins WA, Segraves R, Pinkel D \& Wyrobek AJ 1993 Detection of aneuploid human sperm by fluorescence in situ hybridization: evidence for a donor difference in frequency of sperm disomic for chromosomes 1 and Y. American Journal of Human Genetics 52 799-807.

Robbins WA, Elashoff DA, Xun L, Jia J, Li N, Wu G \& Wei F 2005 Effect of lifestyle exposures on sperm aneuploidy. Cytogenetic and Genome Research 111 371-377. (https://doi.org/10.1159/000086914)

Rodrigo L, Peinado V, Mateu E, Remohi J, Pellicer A, Simon C, Gil-Salom M \& Rubio C 2010 Impact of different patterns of sperm chromosomal abnormalities on the chromosomal constitution of preimplantation embryos. Fertility and Sterility 94 1380-1386. (https://doi.org/10.1016/j. fertnstert.2009.05.061)

Rubes J, Vozdova M, Oracova E \& Perreault SD 2005 Individual variation in the frequency of sperm aneuploidy in humans. Cytogenetic and Genome Research 111 229-236. (https://doi.org/10.1159/000086893)

Rubio C, Gil-Salom M, Simon C, Vidal F, Rodrigo L, Minguez Y, Remohi J \& Pellicer A 2001 Incidence of sperm chromosomal abnormalities in a risk population: relationship with sperm quality and ICSI outcome. Human Reproduction 16 2084-2092. (https://doi.org/10.1093/ humrep/16.10.2084) 
Rubio C, Buendia P, Rodrigo L, Mercader A, Mateu E, Peinado V, Delgado A, Milan M, Mir P, Simon C et al. 2009 Prognostic factors for preimplantation genetic screening in repeated pregnancy loss. Reproductive BioMedicine Online 18 687-693. (https://doi.org/10.1016/ S1472-6483(10)60015-6)

Samplaski MK, Agarwal A, Sharma R \& Sabanegh E 2010 New generation of diagnostic tests for infertility: review of specialized semen tests. International Journal of Urology 17 839-847. (https://doi.org/10.1111/ j.1442-2042.2010.02619.x)

Samuel R, Feng H, Jafek A, Despain D, Jenkins T \& Gale B 2018 Microfluidic-based sperm sorting and analysis for treatment of male infertility. Translational Andrology and Urology 7 S336-S347. (https:// doi.org/10.21037/tau.2018.05.08)

Sanchez-Castro M, Jimenez-Macedo AR, Sandalinas M \& Blanco J 2009 Prognostic value of sperm fluorescence in situ hybridization analysis over PGD. Human Reproduction 1516-1521. (https://doi.org/10.1093/ humrep/dep037)

Shi Q \& Martin RH 2000 Aneuploidy in human sperm: a review of the frequency and distribution of aneuploidy, effects of donor age and lifestyle factors. Cytogenetics and Cell Genetics 90 219-226. (https:// doi.org/10.1159/000056773)

Soares SR, Templado C, Blanco J, Egozcue J \& Vidal F 2001 Numerical chromosome abnormalities in the spermatozoa of the fathers of children with trisomy 21 of paternal origin: generalised tendency to meiotic nondisjunction. Human Genetics 108 134-139. (https://doi.org/10.1007/ s004390000449)

Stern C, Pertile M, Norris H, Hale L \& Baker HW 1999 Chromosome translocations in couples with in-vitro fertilization implantation failure. Human Reproduction 14 2097-2101. (https://doi.org/10.1093/ humrep/14.8.2097)

Sun F, Kozak G, Scott S, Trpkov K, Ko E, Mikhaail-Philips M, Bestor TH, Moens P \& Martin RH 2004a Meiotic defects in a man with nonobstructive azoospermia: case report. Human Reproduction 19 1770-1773. (https://doi.org/10.1093/humrep/deh335)

Sun F, Oliver-Bonet M, Liehr T, Starke H, Ko E, Rademaker A, Navarro J, Benet J \& Martin RH 2004b Human male recombination maps for individual chromosomes. American Journal of Human Genetics 74 521-531. (https://doi.org/10.1086/382138)

Sun F, Trpkov K, Rademaker A, Ko E \& Martin RH 2005 Variation in meiotic recombination frequencies among human males. Human Genetics 116 172-178. (https://doi.org/10.1007/s00439-004-1215-6)

Sun F, Oliver-Bonet M, Liehr T, Starke H, Ko E, Rademaker A \& Martin RH 2007 Discontinuities and unsynapsed regions in meiotic chromosomes have a trans effect on meiotic recombination of some chromosomes in human males. Cytogenetic and Genome Research 119 27-32. (https:// doi.org/10.1159/000109615)

Sun F, Mikhaail-Philips M, Oliver-Bonet M, Ko E, Rademaker A, Turek P \& Martin RH 2008 The relationship between meiotic recombination in human spermatocytes and aneuploidy in sperm. Human Reproduction 23 1691-1697. (https://doi.org/10.1093/humrep/den027)

Tempest HG 2011 Meiotic recombination errors, the origin of sperm aneuploidy and clinical recommendations. Systems Biology in Reproductive Medicine 57 93-101. (https://doi.org/10.3109/19396368 .2010.504879)

Tempest HG \& Griffin DK 2004 The relationship between male infertility and increased levels of sperm disomy. Cytogenetic and Genome Research 107 83-94. (https://doi.org/10.1159/000079575)

Tempest HG, Homa ST, Dalakiouridou M, Christopikou D, Wright D, Zhai XP \& Griffin DK 2004 The association between male infertility and sperm disomy: evidence for variation in disomy levels among individuals and a correlation between particular semen parameters and disomy of specific chromosome pairs. Reproductive Biology and Endocrinology 2 82. (https://doi.org/10.1186/1477-7827-2-82)

Tempest HG, Homa ST, Zhai XP \& Griffin DK 2005 Significant reduction of sperm disomy in six men: effect of traditional Chinese medicine? Asian Journal of Andrology 7 419-425. (https://doi.org/10.1111/j.17457262.2005.00068.x)

Tempest HG, Ko E, Chan P, Robaire B, Rademaker A \& Martin RH 2008 Sperm aneuploidy frequencies analysed before and after chemotherapy in testicular cancer and Hodgkin's lymphoma patients. Human Reproduction 23 251-258. (https://doi.org/10.1093/humrep/dem389)
Tempest HG, Ko E, Rademaker A, Chan P, Robaire B \& Martin RH 2009 Intra-individual and inter-individual variations in sperm aneuploidy frequencies in normal men. Fertility and Sterility 91 185-192. (https:// doi.org/10.1016/j.fertnstert.2007.11.002)

Tempest HG, Cheng SY, Gillott DJ, Handyside AH, Thornhill AR \& Griffin DK 2010 Scoring of sperm chromosomal abnormalities by manual and automated approaches: qualitative and quantitative comparisons. Asian Journal of Andrology 12 257-262. (https://doi. org/10.1038/aja.2009.85)

Templado C, Vidal F \& Estop A 2011 Aneuploidy in human spermatozoa. Cytogenetic and Genome Research 133 91-99. (https://doi. org/10.1159/000323795)

Templado C, Uroz L \& Estop A 2013 New insights on the origin and relevance of aneuploidy in human spermatozoa. Molecular Human Reproduction 19 634-643. (https://doi.org/10.1093/molehr/gat039)

Uroz L \& Templado C 2012 Meiotic non-disjunction mechanisms in human fertile males. Human Reproduction 27 1518-1524. (https://doi. org/10.1093/humrep/des051)

Van Steirteghem A, Bonduelle M, Devroey P \& Liebaers I 2002 Follow-up of children born after ICSI. Human Reproduction Update 8 111-116. (https://doi.org/10.1093/humupd/8.2.111)

Vanneste E, Voet T, Le Caignec C, Ampe M, Konings P, Melotte C, Debrock S, Amyere M, Vikkula M, Schuit F et al. 2009 Chromosome instability is common in human cleavage-stage embryos. Nature Medicine 15 577-583. (https://doi.org/10.1038/nm.1924)

Vera-Rodriguez $M$, Michel CE, Mercader A, Bladon AJ, Rodrigo L, Kokocinski F, Mateu E, Al-Asmar N, Blesa D, Simon C et al. 2016 Distribution patterns of segmental aneuploidies in human blastocysts identified by next-generation sequencing. Fertility and Sterility 105 1047-1055.e1042. (https://doi.org/10.1016/j.fertnstert.2015.12.022)

Voullaire L, Slater H, Williamson R \& Wilton L 2000 Chromosome analysis of blastomeres from human embryos by using comparative genomic hybridization. Human Genetics 106 210-217. (https://doi.org/10.1007/ s004390051030)

Wang L, Zong S, Wang Z, Lu J, Chen C, Zhang R \& Cui Y 2018 Single molecule localization imaging of telomeres and centromeres using fluorescence in situ hybridization and semiconductor quantum dots. Nanotechnology 29 285602. (https://doi.org/10.1088/1361-6528/ aabf72)

Warburton D 1991 De novo balanced chromosome rearrangements and extra marker chromosomes identified at prenatal diagnosis: clinical significance and distribution of breakpoints. American Journal of Human Genetics 49 995-1013.

Webster A \& Schuh M 2017 Mechanisms of aneuploidy in human eggs. Trends in Cell Biology 27 55-68. (https://doi.org/10.1016/j. tcb.2016.09.002)

Wellesley D, Dolk H, Boyd PA, Greenlees R, Haeusler M, Nelen V, Garne E, Khoshnood B, Doray B, Rissmann A et al. 2012 Rare chromosome abnormalities, prevalence and prenatal diagnosis rates from populationbased congenital anomaly registers in Europe. European Journal of Human Genetics 20 521-526. (https://doi.org/10.1038/ejhg.2011.246)

Wells D \& Delhanty JD 2000 Comprehensive chromosomal analysis of human preimplantation embryos using whole genome amplification and single cell comparative genomic hybridization. Molecular Human Reproduction 6 1055-1062. (https://doi.org/10.1093/molehr/6.11.1055)

World Health Organization 2010 WHO Laboratory Manual for the Examination and Processing of Human Semen. World Health Organization.

Wyrobek AJ 1993 Methods and concepts in detecting abnormal reproductive outcomes of paternal origin. Reproductive Toxicology 7 (Supplement 1) 3-16. (https://doi.org/10.1016/0890-6238(93)90064-E)

Xing C, Marchetti F, Li G, Weldon RH, Kurtovich E, Young S, Schmid TE, Zhang L, Rappaport S, Waidyanatha S et al. 2010 Benzene exposure near the U.S. permissible limit is associated with sperm aneuploidy. Environmental Health Perspectives 118 833-839. (https://doi. org/10.1289/ehp.0901531)

Yatsenko AN \& Turek PJ 2018 Reproductive genetics and the aging male. Journal of Assisted Reproduction and Genetics 35 933-941. (https://doi. org/10.1007/s10815-018-1148-y)

Yusuf M, Bauer DL, Lipinski DM, Maclaren RE, Wade-Martins R, Mir KU \& Volpi EV 2011 Combining M-FISH and Quantum Dot technology for fast 
chromosomal assignment of transgenic insertions. BMC Biotechnology 11 121. (https://doi.org/10.1186/1472-6750-11-121)

Zalensky A \& Zalenskaya I 2007 Organization of chromosomes in spermatozoa: an additional layer of epigenetic information? Biochemical Society Transactions 35 609-611. (https://doi.org/10.1042/BST0350609)

Zhang MH, Zhai LP, Fang ZY, Li AN, Qiu Y \& Liu YX 2018 Impact of a mild scrotal heating on sperm chromosomal abnormality, acrosin activity and seminal alpha-glucosidase in human fertile males. Andrologia $\mathbf{5 0}$ e12985. (https://doi.org/10.1111/and.12985)

Zidi-Jrah I, Hajlaoui A, Mougou-Zerelli S, Kammoun M, Meniaoui I, Sallem A, Brahem S, Fekih M, Bibi M, Saad A et al. 2016 Relationship between sperm aneuploidy, sperm DNA integrity, chromatin packaging, traditional semen parameters, and recurrent pregnancy loss. Fertility and Sterility 105 58-64. (https://doi.org/10.1016/j.fertnstert.2015.09.041)

Received 21 June 2018

First decision 23 July 2018

Revised manuscript received 28 October 2018

Accepted 1 November 2018 